OPEN ACCESS

Edited by:

Jorg Hermann Fritz,

McGill University, Canada

Reviewed by:

Michaela Gack,

The University of Chicago, USA

Angela Battistini,

Istituto superiore di sanita, Italy

*Correspondence:

Karen L. Mossman

mossk@mcmaster.ca

Specialty section:

This article was submitted to Molecular Innate Immunity,

a section of the journal

Frontiers in Immunology

Received: 14 September 2016

Accepted: 26 October 2016

Published: 11 November 2016

Citation:

Schulz KS and Mossman KL (2016) Viral Evasion Strategies in Type I IFN Signaling - A Summary

of Recent Developments.

Front. Immunol. 7:498. doi: 10.3389/fimmu.2016.00498

\section{Viral Evasion Strategies in Type I IFN Signaling - A Summary of Recent Developments}

\author{
Katharina S. Schulz and Karen L. Mossman* \\ Department of Pathology and Molecular Medicine, McMaster Immunology Research Centre, Institute for Infectious Disease \\ Research, McMaster University, Hamilton, ON, Canada
}

The immune system protects the organism against infections and the damage associated with them. The first line of defense against pathogens is the innate immune response. In the case of a viral infection, it induces the interferon (IFN) signaling cascade and eventually the expression of type I IFN, which then causes an antiviral state in the cells. However, many viruses have developed strategies to counteract this mechanism and prevent the production of IFN. In order to modulate or inhibit the IFN signaling cascade in their favor, viruses have found ways to interfere at every single step of the cascade, for example, by inducing protein degradation or cleavage, or by mediate protein polyubiquitination. In this article, we will review examples of viruses that modulate the IFN response and describe the mechanisms they use.

Keywords: virus, type I interferon, evasion, innate immune signaling, NFкB

\section{INTRODUCTION}

The mammalian immune system evolved to detect and fight viral infections effectively. The induction of type I interferon (IFN), predominantly IFN- $\alpha$ and IFN- $\beta$, forms the first line of defense. The type I IFN response consists of two parts. First, the cell produces type I IFN, when triggered by a viral stimulus. The IFN is then secreted and, in the second part of the response, it is sensed by the producing, as well as neighboring cells, resulting in the production of IFN-stimulated genes (ISGs) [reviewed in Ref. (1)].

Viruses, which have coevolved with their host, develop strategies to counteract the signaling cascades of the innate immune system and ensure their replication. Recently, several reviews were published, describing the innate immune evasion strategies of individual viruses or virus families, such as influenza virus (2,3), Phleboviruses (4), Herpes viruses (5-7), Coronaviruses severe acute respiratory syndrome (SARS) and middle east respiratory syndrome (MERS) (8), human immunodeficiency virus (HIV) $(9,10)$, as well as multiple RNA viruses $(11,12)$. Moreover, there are recent articles that review how viruses prevent detection by pathogen recognition receptors (PRRs) $(13,14)$ and how viruses modulate innate immune signaling by use of viral deubiquitinases (15).

In this review, we will compare the different strategies viruses have developed to suppress innate immune signaling of individual components of the innate immune signaling cascade. Due to the tremendous amount of data in this field, we will focus on recent discoveries. Older studies were summarized in Ref. $(16,17)$. 


\section{VIRUS RECOGNITION}

Invading viruses are recognized by PRRs [reviewed in Ref. (14)]. The most important viral markers for the innate immune system are viral nucleic acids. The detection of viral DNA through the cGAS-Sting pathway and the counter measurements taken by viruses have been reviewed recently (18) and are not part of this review.

Viral RNAs, which are mostly double-stranded (ds-)RNA, are recognized by three PRRs: the endosomal toll-like receptor 3 (TLR3), the cytoplasmic retinoic acid-inducible gene I (RIG-I)like receptors (RLRs), and the nucleotide-oligomerization domain (NOD)-like receptors (NLRs) (19). TLR3 and the RLRs are important for inducing the type I IFN response, whereas NLRs have been shown to regulate interleukin-1 $\beta$ (IL-1 $\beta$ ) maturation through activation of caspase-1 (20). The group of RLRs consists of RIG-I, melanoma differentiation-associated gene 5 (MDA5), and laboratory of genetics and physiology 2 (LGP2). The three receptors have a similar structure, all containing a caboxyterminal domain, which functions as a repressor domain (RD) in RIG-I and LGP2 (21) and a central helicase domain, but LGP2 lacks the caspase activation and recruitment domains (CARDs) that function in signaling [reviewed in Ref. $(19,22)]$. Both the helicase and the carboxy-terminal domain are required for RNA binding. RIG-1 and MDA-5 detect specific viral RNA PAMPs, while LGP2 negatively regulates RIG-I signaling and promotes RNA binding to MDA5 [reviewed in detail in Ref. (14)].

In unstimulated cells, RIG-I and MDA-5 are kept in a repressed state due to phosphorylations on serine and threonine residues in the CARDs and carboxy-terminal domains $(23,24)$. Upon binding of RNA, both RIG-I and MDA-5 undergo conformational changes, resulting in release of their CARDs $(25,26)$. Recruited phosphatases remove the phosphate residues, and E3 ubiquitin ligases attach Lys63-linked ubiquitin polymers onto the CARDs and C-terminal domain of RIG-I, which are important for RIG-I tetramerization (27-31).

RNA-bound RIG-1 then interacts with 14-3-3e, a mitochondrial trafficking protein, and the TRIM25 ubiquitin ligase, which together transport RIG-I to the mitochondria (32). There the CARDs of RIG-I or MDA-5 interact with the CARD of the mitochondrial activator of virus signaling (MAVS, also known as IPS-1, VISA, and Cardif), which is an essential signaling adaptor protein. The activation of MAVS has recently been reviewed in detail in Ref. (33).

TLR3 interacts with TRIF, which serves as a molecular platform and forms physical interactions with several adaptor molecules (34). By interacting with upstream adaptors, TRIF undergoes conformational changes and recruits the downstream TNF receptor-associated factor (TRAF) 3 and TRAF6 [reviewed in Ref. (35)]. The kinase receptor-interacting protein-1 (RIP-1) is part of both the signaling pathways downstream of TLR3 and RIG-I. It can interact with TRIF to induce NFkB activation (36). Moreover, the dsRNA-activated TLR3 can recruit TRIF, RIP-1, and Caspase- 8 and induce apoptosis (37). Also, RIP-1 and its adaptor protein Fas-associated protein with death domain (FADD) are part of the signaling cascade downstream of RIG-I and MDA-5 and involved in the activation of the transcription factors interferon regulatory factor (IRF) 3 and IRF7 (38). TRAF3 serves as a linker between the upstream adaptor proteins (TRIF or MyD88 for TLRs and MAVS for RLRs) and the downstream signaling kinases TBK1/IKK $\varepsilon$ or IRAK1/IKK $\alpha$. The recruitment of TRAF3 to the TLR or RLR signaling complexes activates the E3 ligase activity of TRAF3, which then catalyzes its own K63-linked ubiquitinylation. Subsequent TRAF3 activates TBK1/IKKe or IRAK/IKK $\alpha$ [reviewed in Ref. (39)] (Figure 1).

Viruses target RIG-I directly or indirectly to block the type I IFN response. The phlebovirus Toscana Virus expresses a non-structural protein, which directly interacts with RIG-I and induces its proteasomal degradation $(40,41)$. Foot-and-mouth disease virus (FMDV) proteins $\mathrm{L}^{\text {pro }}, 3 \mathrm{C}^{\text {pro }}$, and $2 \mathrm{~B}$ increase the RIG-I mRNA expression but decrease the protein expression of RIG-I. L $^{\text {pro }}$ and $3 \mathrm{C}^{\text {pro }}$ both induce RIG-I degradation, whereas the mechanism of how 2B reduces RIG-I protein levels has not been solved yet (42). Other viruses target RIG-I indirectly. Hepatitis B virus (HBV) induces miR146a, which then posttranscriptionally inhibits the expression of RIG-I and suppresses the production of type I IFN (43).

The dengue virus NS3 protein binds to $14-3-3 \varepsilon$ and prevents the translocation of RIG-I to MAVS. The binding site on NS3 is a highly conserved phosphomimetic motif, which was verified by generation of a virus containing a mutation in this motif (44).

It has been proposed that in certain cell types RIG-I requires sentinels, such as the protein DDX60, which associates with RIG-I and promotes the RIG-I RNA-binding activity $(45,46)$. Other studies question DDX60 acting as a broadly active enhancer of antiviral responses $(47,48)$ and instead suggest that DDX60 only functions in the antiviral response to specific viruses, such as hepatitis $\mathrm{C}$ virus (47). However, there are data indicating that influenza $A$ virus and hepatitis $C$ virus attenuate IFN $\beta$-promoter activation by targeting the sentinel DDX60. Both viruses activate the epidermal growth factor (EGF) receptor, which in turn phosphorylates DDX60 on Tyr-793 and Tyr-796. This results in the attenuation of DDX60-dependent RIG-I activation. In addition, independent of its role as sentinel for RIG-I viral RNA recognition, DDX60 plays a role in viral RNA degradation (46) (Figure 1).

Mitochondrial activator of virus signaling is blocked by different viruses in various ways. The dengue virus protein NS4A targets MAVS, and the interaction prevents the binding of MAVS to RIG-I (49). The porcine reproductive and respiratory syndrome virus (PRRSV) 3C-like protease (3CLSP), by contrast, cleaves MAVS in a proteasome- and caspase-independent manner at Glu268 (E268/G269). Both cleavage products fail to activate the type I IFN response (50). Likewise, the hepatitis $C$ virus protein NS3-4A $(51,52)$, as well as the highly pathogenic porcine reproductive and respiratory syndrome virus (HP-PRRSV) protein nsp4 (53) have been shown to cleave MAVS and block RLR signaling. The porcine epidemic diarrhea virus (PEDV) also targets MAVS in small intestinal epithelial cells (IECs). However, the exact mechanism has not been solved yet (54) (Figure 1).

The SARS coronavirus protein ORF9b not only influences antiviral signaling but also alters host cell mitochondria morphology by inducing degradation of the dynamin-like protein (DRP1). MAVS becomes concentrated into small puncta in the presence 


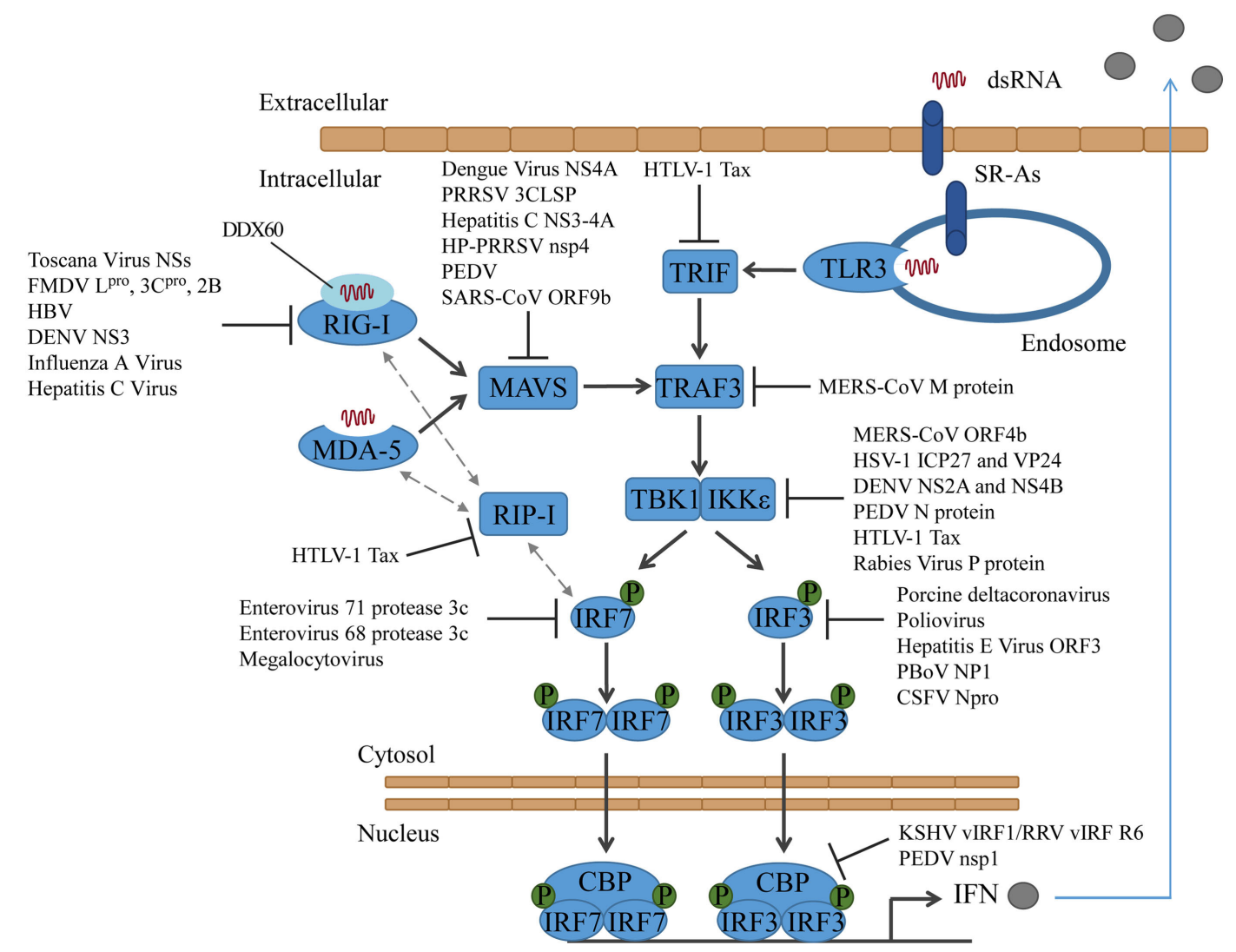

FIGURE 1 | Activation of interferon regulatory factors and the counteractions taken by viruses. DsRNA is sensed by PRRs, which results in the activation of different adaptor proteins and the recruitment of TRAF3. TBK1 and/or IKKE are activated and phosphorylate IRF3 and/or IRF7, which then translocate into the nucleus to induce type I IFN expression. CSFV, classical swine fever virus; DENV, dengue virus; FMDV, foot-and-mouth disease virus; HBV, hepatitis B virus; HP-PRRSV, highly pathogenic porcine reproductive and respiratory syndrome virus; HSV-1, herpes simplex virus type 1; HTLV-1, human T-cell lymphotropic virus type I; KSHV, Kaposi's sarcoma-associated herpesvirus; MERS-CoV, middle east respiratory syndrome coronavirus; PBoV, porcine bocavirus; PEDV, porcine epidemic diarrhea virus; PRRSV, porcine reproductive and respiratory syndrome virus; RRV, rhesus macaque rhadinovirus.

of ORF9b (55). In addition, ORF9b triggers K48-linked ubiquitinylation of MAVS, by targeting the poly $(\mathrm{C})$-binding protein 2 (PCBP2) and the HECT domain E3 ligase AIP4. Under normal conditions, PCBP2 controls MAVS levels by linking the AID4 E3 ubiquitin ligase with MAVS (56). In addition to MAVS, also the levels of TRAF3 and TRAF6 are reduced by ORF9b. However, it is unlikely that TRAF3 and TRAF6 are targeted directly. More likely, they are degraded due to their interaction with MAVS (55) (Figure 1).

Human T-cell lymphotropic virus type I (HTLV-1) protein Tax disrupts innate immune signaling in multiple ways: it binds to the RIP homotypic interaction motif (RHIM) domains of RIP-1 and disrupts the interaction between RIP-1 and RIG-I or MDA-5 and the activation of the type I IFN promoter. Tax also binds to TRIF and thereby interrupts the TLR3 signaling cascade. Finally, Tax blocks the association between RIP-1 and IRF7, which resulted in repression of the IRF7 activity (57) (Figure 1).

Middle East respiratory syndrome coronavirus $M$ protein interacts with TRAF3 and disrupts the interaction between TRAF3 and TBK1, which ultimately leads to a reduced IRF3 activation. For the interaction with TRAF3, the N-terminal transmembrane domain of the MERS-CoV M protein is sufficient (58), similar to what has been shown for SARS-CoV before (59) (Figure 1).

\section{ACTIVATION OF TRANSCRIPTION FACTORS AND IFN TRANSCRIPTION}

Triggering of the TLR3- and RLR-signaling cascade results in the activation of the transcription factors NFKB and IRF3/IRF7. In its inactive state, the transcription factor NFKB is complexed with its

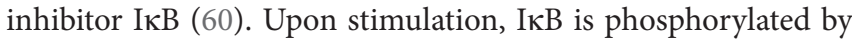
the I $\mathrm{B}$ k kinase (IKK) complex, which is composed of two catalytic subunits, such as IKK $\alpha$ and IKK $\beta$, and a regulatory subunit, such as NFKB essential modulator (NEMO) (61). The phosphorylation of $\mathrm{I} \kappa \mathrm{B} \alpha$ induces its polyubiquitination through the E3 ubiquitin ligase $\beta$-transducin repeat-containing protein $(\beta-\operatorname{TrCP})$ and subsequent proteasomal degradation (62), allowing $\mathrm{NF \kappa B}$ to translocate into the nucleus and induce the expression of target genes (63) (Figure 2). 


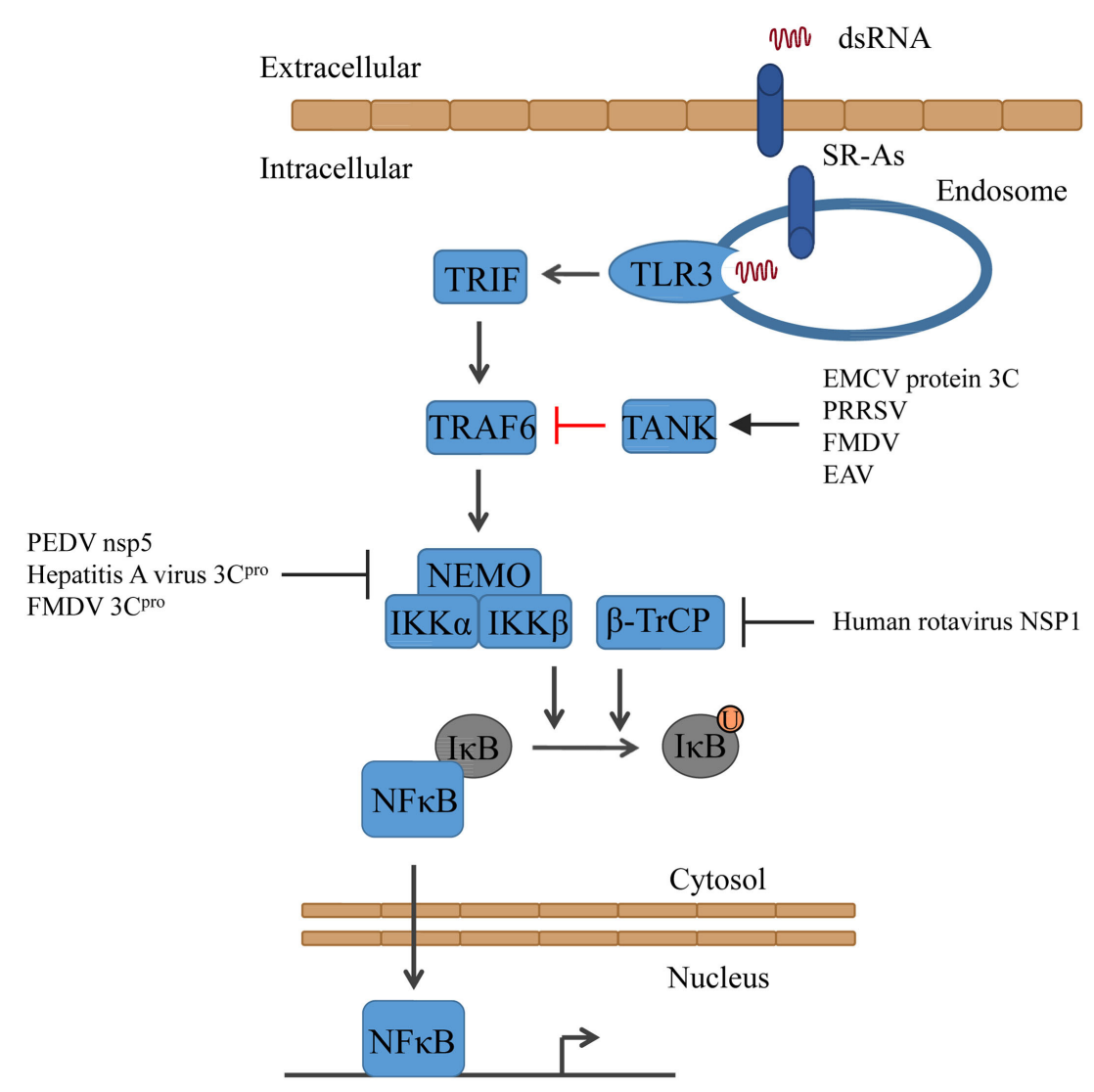

FIGURE 2 Activation of NFKB signaling and the counteractions taken by viruses. Triggering of TLR3 results in the activation of first TRAF6 and subsequently of IKK (consisting of NEMO, IKK $\alpha$, and IKK $\beta$ ). Together with $\beta$ - TrCP, IKK mediates the ubiquitinylation of IKB, resulting in the release of NFKB. EAV, equine arteritis virus; EMCV, encephalomyocarditis virus; FMDV, foot-and-mouth diseases virus; PEDV, porcine epidemic diarrhea virus; PRRSV, porcine reproductive and respiratory syndrome virus.

Encephalomyocarditis virus (EMCV) protein 3C cleaves TRAF family member-associated NFkB activator (TANK), which inhibits TRAF6-mediated NFKB activation, on Gln291. As a result, $\mathrm{NFKB}$ is activated and the unstable $\mathrm{C}$-terminal fragment of TANK is subjected to proteasomal degradation (64). Also, other viruses express proteases that cleave TANK, although on other residues, such as porcine reproductive and respiratory syndrome virus (PRRSV) (TANK is cleaved by Nsp4), FMDV (protease $3 \mathrm{C}$ cleaves TANK), and equine arteritis virus (EAV) (TANK is cleaved by Nsp4). Thus, TANK seems to be a common target of several positive RNA viral proteases (64) (Figure 2).

Several viruses have been shown to disrupt IFN signaling by cleaving NEMO. PEDV 3C-like protease, nsp5, cleaves NEMO at $\mathrm{Gln} 231$ (65), whereas the hepatitis A virus $3 \mathrm{C}$ protease (3C $\mathrm{C}^{\mathrm{pro}}$ ) cleaves NEMO at Gln304 (66) and the picornavirus FMDV protease $3 \mathrm{C}^{\text {pro }}$ at $\mathrm{Gln} 383$, removing the C-terminal zinc finger domain from the protein (67). The human rotavirus has developed another way. Its non-structural protein 1 (NSP1) has been shown to inhibit the NFkB pathway by degrading $\beta$-TrCP and consequently stabilizing IKB (68) (Figure 2).

TANK-binding kinase 1 (TBK1) and inhibitor of $\kappa B$ kinase $\varepsilon$ (IKKe) are classified as non-canonical serine/threonine kinases and are both able to induce IRF3 and IRF7 phosphorylation and subsequent dimerization (69-72). However, while TBK1 is constitutively expressed in most cell types, the expression of IKKe is more restricted (73). Upon stimulation, TBK1 and IKKe are recruited by adaptor proteins to signaling complexes to be activated by phosphorylation on Ser172 and both have been shown to be subjected to K63-linked polyubiquitination [reviewed in Ref. $(73,74)]$. For TBK1, K63-linked polyubiquitination seems to be important for TLR- and RLR-induced IFN production, as ubiquitin chains might serve as a platform for the assembly of TBK1 signaling complexes. Moreover, deubiquitinases are able to terminate the TBK1-mediated pathway by cleaving the K63linked ubiquitin chains [reviewed in Ref. $(74,75)]$. Activated TBK1/IKKe phosphorylates IRF3 and/or IRF7 in the cytosol at specific serine residues. This phosphorylation results in homo- or heterodimerization of IRF3 and IRF7 and nuclear translocation $(76,77)$. Interestingly, while IRF3 is constitutively expressed, IRF7 is expressed at low levels in most cell types and expression is induced upon IFN signaling. Therefore, in most cells, IRF7 strongly enhances the production of IFN [reviewed in Ref. (78)]. Once phosphorylated IRF3 and/or IRF7 dimers have translocated into the nucleus, they bind to the transcription coactivator 
CREB-binding protein $(\mathrm{CPB}) / \mathrm{p} 300(79,80)$. Together with other factors, such as NFKB, they form the enhanceosome on the IFN $\beta$ promoter and induce the expression of type I IFN [reviewed in Ref. (76)].

The viral proteins that target TBK1 act by either blocking activation of TBK1 by MAVS or by inhibiting activation of IRF3 by TBK1. The MERS-CoV protein ORF4b blocks IFN $\beta$ production by binding to TBK1 and IKKe and suppressing the formation of a MAVS/IKKe complex (81). In addition to inhibiting TBK1/IKKe activation, ORF4b can also inhibit the production of IFN $\beta$ in the nucleus; however, the mechanism has not been solved yet (81). Recently, two herpes simplex virus proteins have been shown to target TBK1/IKK $\varepsilon$ and inhibit the phosphorylation of IRF3: ICP27 (82) and VP24 (83). Also, dengue virus serotype 4 non-structural proteins NS2A and NS4B, as well as the NS2A and NS4B proteins of other Dengue viruses, inhibit the phosphorylation of TBK1 (84) and PEDV N protein has been shown to interact with TBK1, hampering the association of TBK1 with IRF3 and preventing the activation of IRF3 activation (85). The human T-cell leukemia virus type 1 oncoprotein Tax has been shown to also interact with TBK1. However, studies came to contradicting results on how that influences the production of IFN $\beta$. While one group showed that Tax activates TBK1 and the production of IFN $\beta$ (86), another group showed that Tax suppresses the IFN production by interaction with TBK1 (87). Interestingly, when a recent study tested how the rabies virus $P$ protein of street strains behaves compared to laboratory-adapted strains with regard to the induction of type I IFN, they found that both street strains and laboratory strains inhibit TBK1-mediated signaling, but only the P protein of street strains also interacts with and inhibits IKKe-inducible IRF3dependent IFN $\beta$ expression (88) (Figure 1).

Interferon regulatory factor 3 is targeted by many viruses to impair innate immune signaling. Most viruses inhibit the phosphorylation and thereby also the dimerization and translocation of IRF3, such as the porcine deltacoronavirus (89) or poliovirus (90). Hepatitis E virus protein ORF3 also suppresses IRF3 phosphorylation, but in an indirect way. It activates the signal regulator protein $\alpha$ (SIRP- $\alpha$ ), which negatively regulates type I IFN induction (91). In contrast, porcine bocavirus (PBoV) NP1 protein does not affect IRF3 expression, phosphorylation, or nuclear translocation. Instead, it interacts with the DNA-binding domain of IRF3 and inhibits the DNA-binding activity (92). A very interesting way of how to circumvent the host innate immune response was found when studying gammaherpesviruses Kaposi's sarcoma-associated herpesvirus (KSHV) and rhesus macaque rhadinovirus (RRV). They express several viral homologs to the IRFs, called viral IRFs (vIRFs). These vIRFs have found multiple ways to suppress type I IFN production. For KSHV, different strategies have been reviewed in Ref. (6). Recently, the RRV vIRF R6 has been shown to interact with the transcriptional coactivator CBP in the nucleus, similar to the KSHV vIRF1. As a result, CBP cannot form a complex with the phosphorylated IRF3, and the IFN expression is not induced (93-95). Interestingly, RRV R6 is the first vIRF for which an association with the viron could be shown. Therefore, vIRF V6 can shut down the type I IFN response shortly after the cell was infected, rendering the cell more susceptible to infection (95). The PEDV protein nsp1 also targets
CBP. Nsp1 induces CBP degradation in a proteasome-dependent manner and thereby interrupts enhanceosome assembly and the production of type I IFN (96) (Figure 1).

For most of these interactions, the molecular mechanisms have not been unraveled yet. A protein that has been shown to interact with and induce proteasomal degradation of IRF3 some time ago is classical swine fever virus (CSFV) Npro (97, 98). Recently, the molecular mechanism has been published. IRF3 and Npro interact direct and form a soluble 1:1 complex. Moreover, it was shown that Npro interacts with the full-length IRF3, not with individual domains, and that Npro binds the constitutively active form of IRF3 in the presence of CPB. Thus, Npro interacts with both the monomer and the active IRF3 dimer and likely targets both species for ubiquitinylation and proteasomal degradation (99).

Interferon regulatory factor 7 is targeted by two human enteroviruses, such as enterovirus 71 and enterovirus 68 . They downregulate IRF7 by cleaving it with their protease $3 c$, leaving the cleavage products unable to induce IFN expression. While enterovirus 71 cleaves IRF7 once at Gln189-Ser190 (100), Enterovirus 68 cleaves it twice, the cleavage sites being Gln167 and Gln189 (101). Moreover, megalocytivirus, a DNA virus that infects marine and freshwater fish, induces the expression of the host microRNA pol-miR-731, which then specifically suppresses the expression of IRF7 (102) (Figure 1).

\section{TYPE I IFN SIGNALING}

The type I IFNs act in an autocrine, paracrine, or systemic manner to stimulate antiviral responses. They are recognized by the IFN $\alpha / \beta$ receptor (IFNAR), which consists of the subunits IFNAR1 and IFNAR2 expressed on virtually all cell types (103). The interaction of type I IFN with the receptor results in the phosphorylation and activation of the IFNAR1- and IFNAR2-associated tyrosine kinases tyrosine kinase 2 (TYK2) and Janus kinase 1 (JAK1), which then phosphorylate IFNAR tyrosine residues, resulting in the recruitment and activation of signaling molecules, such as the signal transducer and activator of transcription (STAT) family of transcription factors $(104,105)$. Upon activation, STAT1 and STAT2, together with IRF9, form the IFN-stimulated gene factor 3 (ISGF3), which then translocates into the nucleus to induce transcription of ISGs [reviewed in detail in Ref. (106-108)].

Several viruses target IFNAR to prohibit IFN binding and signaling. Influenza virus induces the degradation of IFNAR1. Hemagglutinin (HA) triggers the phosphorylation and ubiquitinylation of IFNAR1, thus promoting protein degradation (109). Encephalitic Flaviviruses, such as tick-borne encephalitis virus or West Nile virus, inhibit IFNAR1 surface expression. Their protein NS5 binds the cellular dipeptidase prolidase (PEPD), which is involved in IFNAR1 maturation and accumulation, activation of IFN $\beta$-stimulated gene induction, and IFN-dependent viral control. This interaction inhibits IFNAR1 intracellular trafficking and glycosylation but does not promote IFNAR1 degradation (110) (Figure 3).

Both STAT1 and STAT2 are targeted by many viruses to suppress ISG induction. PEDV induces Stat1 ubiquitinylation and targets it for degradation in the proteasomes (111). 


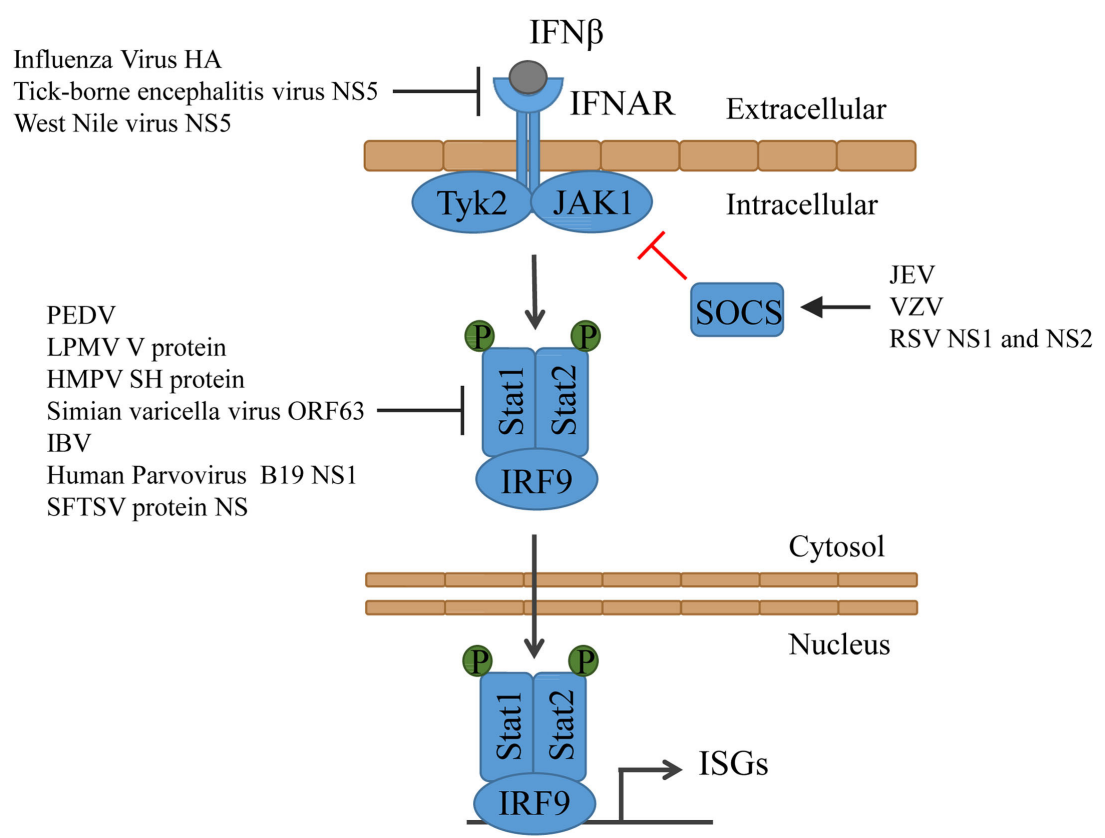

FIGURE 3 | Type I IFN signaling and the counteractions taken by viruses. IFN binds to its receptor and thereby activates Tyk2 and Jak1, which then phosphorylate Stat1 and Stat2. Together with IRF9, Stat1 and Stat2 form the ISGF3, which translocates into the nucleus and induces the expression of ISGs. HMPV, human metapneumovirus; IBV, infectious bronchitis virus; JEV, Japanese encephalitic virus; LPMV, La Piedad Michoacán Mexico Virus; PEDV, porcine epidemic diarrhea virus; RSV, respiratory syncytial virus; SFTSV, severe fever with thrombocytopenia syndrome virus; VZV, varicella-zoster virus.

Some viruses evolved to prevent the phosphorylation of Stat1 or Stat2. The paramyxovirus La Piedad Michoacán Mexico Virus (LPMV) V protein binds to Stat2 and prevents the type I IFN-dependent phosphorylation and nuclear translocation of Stat 1 and Stat2 (112). Similarly, human metapneumovirus (HMPV) protein SH impairs Stat1 expression, phosphorylation, and activation (113). Simian varicella virus not only inhibits Stat2 phosphorylation but also promotes degradation of IRF9 in a proteasome-dependent manner through its protein ORF63 (114). Also, infectious bronchitis virus (IBV) inhibits phosphorylation and nuclear translocation of Stat. However, despite detailed analyses, it is unclear which viral protein is responsible. It was, however, shown that the accessory protein 3 a contributes to IBV resistance to type I IFN, although the target is unknown as well (115). In case of the human Parvovirus B19, it becomes evidently clear that both the virus and the immune system constantly evolve to prevail. While its protein NS1 suppresses Stat phosphorylation, the immune system senses the protein and triggers the production of type I IFN (116). SFTSV, an emerging tick-borne pathogen, developed multiple ways to prevent ISG induction. The viral non-structural protein NS impairs Stat1 expression, phosphorylation, and activation (117) and interacts with STAT2 and sequesters STAT1 and STAT2 into viral inclusion bodies, where they are trapped (118) (Figure 3).

The JAK-STAT signal transduction pathway is negatively regulated by the suppressor of cytokine signaling (SOCS) family of proteins in form of a classical feedback loop $(119,120)$. Some viruses induce the expression of SOCS to take advantage of this mechanism to minimize the induction of ISGs. Japanese encephalitic virus (JEV) downregulates the expression of microRNA miR-432, which then results in upregulated SOCS5 levels (121). Varicella-zoster virus (VZV) infection induces the expression of SOCS3 (122) and respiratory syncytial virus (RSV) nonstructural proteins NS1 and NS2 induce upregulation of SOCS1 and SOCS3, which also inhibited the induction of chemokines (123) (Figure 3).

\section{HOST SHUT OFF}

Viruses fully depend on the translation machinery of the host cell for replication. Accordingly, they have evolved multiple ways to hamper host protein synthesis [reviewed in Ref. (124)]. One way is to shut off host protein synthesis. For some time, it was thought that Gamma- and Deltacoronaviruses do not induce host shutoff, such as Alpha- and Betacoronaviruses do. However, a recent study showed that the infectious bronchitis Gammacoronavirus induces host shutoff using its protein $5 \mathrm{~b}$. It seems like $5 \mathrm{~b}$ is a functional equivalent of nsp1, the host shutoff protein of Alpha- and Betacoronaviruses (125).

\section{CONCLUSION}

Viruses evolved to have various strategies to circumvent the innate immune response by blocking the production of type I IFN or the expression of ISGs. While these diverse strategies may appear contradictory between viruses, several factors require consideration. For example, the use of clinical isolates versus 
laboratory-passaged strains could yield different results, particularly with RNA viruses that rapidly accumulate mutations due to error-prone RNA-dependent RNA polymerases. Moreover, the choice of cell line can greatly influence experimental outcomes, as many immortalized or transformed continual cell lines harbor mutations in critical innate immune signaling (126). Likewise, the use of genetic knockout versus knockdown cell lines or organisms can influence experimental outcomes, as can the experimental procedures themselves, particularly when endogenous interactions are disrupted with the use of overexpression approaches.

Studying the mechanisms used by viruses to prevent an immune response is of great importance for the development of new strategies to limit the sequelae of viral infections. Identification of key immune evasion proteins allows development of antivirals to target these proteins. Alternatively, identification of key cellular antiviral pathways allows development

\section{REFERENCES}

1. Nagarajan U. Induction and function of IFNbeta during viral and bacterial infection. Crit Rev Immunol (2011) 31(6):459-74. doi:10.1615/ CritRevImmunol.v31.i6.20

2. Krug RM. Functions of the influenza A virus NS1 protein in antiviral defense. Curr Opin Virol (2015) 12:1-6. doi:10.1016/j.coviro.2015.01.007

3. Weber-Gerlach M, Weber F. To conquer the host, influenza virus is packing it in: interferon-antagonistic strategies beyond NS1. J Virol (2016) 90(19):8389-94. doi:10.1128/JVI.00041-16

4. Wuerth JD, Weber F. Phleboviruses and the type I interferon response. Viruses (2016) 8(6):174-90. doi:10.3390/v8060174

5. Amsler L, Verweij MC, DeFilippis VR. The tiers and dimensions of evasion of the type I interferon response by human cytomegalovirus. J Mol Biol (2013) 425(24):4857-71. doi:10.1016/j.jmb.2013.08.023

6. Kumari P, Narayanan S, Kumar H. Herpesviruses: interfering innate immunity by targeting viral sensing and interferon pathways. Rev Med Virol (2015) 25(3):187-201. doi:10.1002/rmv.1836

7. Su C, Zhan G, Zheng C. Evasion of host antiviral innate immunity by HSV-1, an update. Virol J (2016) 13:38. doi:10.1186/s12985-016-0495-5

8. Vijay R, Perlman S. Middle East respiratory syndrome and severe acute respiratory syndrome. Curr Opin Virol (2016) 16:70-6. doi:10.1016/j.coviro. 2016.01.011

9. Rustagi A, Gale M Jr. Innate antiviral immune signaling, viral evasion and modulation by HIV-1. J Mol Biol (2014) 426(6):1161-77. doi:10.1016/j. jmb.2013.12.003

10. Sauter D, Kirchhoff F. HIV replication: a game of hide and sense. Curr Opin HIV AIDS (2016) 11(2):173-81. doi:10.1097/COH.0000000000000233

11. Zinzula L, Tramontano E. Strategies of highly pathogenic RNA viruses to block dsRNA detection by RIG-I-like receptors: hide, mask, hit. Antiviral Res (2013) 100(3):615-35. doi:10.1016/j.antiviral.2013.10.002

12. Chatterjee S, Basler CF, Amarasinghe GK, Leung DW. Molecular mechanisms of innate immune inhibition by non-segmented negative-sense RNA viruses. J Mol Biol (2016) 428(17):3467-82. doi:10.1016/j.jmb.2016. 07.017

13. Goubau D, Deddouche S, Reis e Sousa C. Cytosolic sensing of viruses. Immunity (2013) 38(5):855-69. doi:10.1016/j.immuni.2013.05.007

14. Chan YK, Gack MU. Viral evasion of intracellular DNA and RNA sensing. Nat Rev Microbiol (2016) 14(6):360-73. doi:10.1038/nrmicro. 2016.45

15. Lin D, Zhong B. Regulation of cellular innate antiviral signaling by ubiquitin modification. Acta Biochim Biophys Sin (Shanghai) (2015) 47(3):149-55. doi:10.1093/abbs/gmu133

16. Taylor KE, Mossman KL. Recent advances in understanding viral evasion of type I interferon. Immunology (2013) 138(3):190-7. doi:10.1111/ imm. 12038 of strategies to enhance these pathways to overwhelm incoming viruses. Information on key immune evasion factors further facilitates the engineering of safe and effective vaccine strains and designing strategies to target new emerging viruses from the same or closely related family.

\section{AUTHOR CONTRIBUTIONS}

KS and KM conceptualized the scope of the review article. KS wrote the review with input from KM.

\section{FUNDING}

This work was supported by a postdoctoral fellowship from the Deutsche Forschungsgemeinschaft (SCHU3011/1-1). Work in the Mossman laboratory on innate antiviral signaling is supported by the Canadian Institutes for Health Research.

17. Devasthanam AS. Mechanisms underlying the inhibition of interferon signaling by viruses. Virulence (2014) 5(2):270-7. doi:10.4161/viru.27902

18. Ma Z, Damania B. The cGAS-STING defense pathway and its counteraction by viruses. Cell Host Microbe (2016) 19(2):150-8. doi:10.1016/j. chom.2016.01.010

19. Kell AM, Gale M Jr. RIG-I in RNA virus recognition. Virology (2015) 47(9-480):110-21. doi:10.1016/j.virol.2015.02.017

20. Kanneganti TD, Lamkanfi M, Nunez G. Intracellular NOD-like receptors in host defense and disease. Immunity (2007) 27(4):549-59. doi:10.1016/j. immuni.2007.10.002

21. Saito T, Hirai R, Loo YM, Owen D, Johnson CL, Sinha SC, et al. Regulation of innate antiviral defenses through a shared repressor domain in RIG-I and LGP2. Proc Natl Acad Sci U S A (2007) 104(2):582-7. doi:10.1073/ pnas.0606699104

22. Fitzgerald ME, Rawling DC, Vela A, Pyle AM. An evolving arsenal: viral RNA detection by RIG-I-like receptors. Curr Opin Microbiol (2014) 20:76-81. doi:10.1016/j.mib.2014.05.004

23. Gack MU, Nistal-Villan E, Inn KS, Garcia-Sastre A, Jung JU. Phosphorylationmediated negative regulation of RIG-I antiviral activity. J Virol (2010) 84(7):3220-9. doi:10.1128/JVI.02241-09

24. Sun Z, Ren H, Liu Y, Teeling JL, Gu J. Phosphorylation of RIG-I by casein kinase II inhibits its antiviral response. J Virol (2011) 85(2):1036-47. doi:10.1128/JVI.01734-10

25. Kowalinski E, Lunardi T, McCarthy AA, Louber J, Brunel J, Grigorov B, et al. Structural basis for the activation of innate immune pattern-recognition receptor RIG-I by viral RNA. Cell (2011) 147(2):423-35. doi:10.1016/j. cell.2011.09.039

26. Kolakofsky D, Kowalinski E, Cusack S. A structure-based model of RIG-I activation. RNA (2012) 18(12):2118-27. doi:10.1261/rna.035949.112

27. Gack MU, Shin YC, Joo CH, Urano T, Liang C, Sun L, et al. TRIM25 RINGfinger E3 ubiquitin ligase is essential for RIG-I-mediated antiviral activity. Nature (2007) 446(7138):916-20. doi:10.1038/nature05732

28. Zeng W, Sun L, Jiang X, Chen X, Hou F, Adhikari A, et al. Reconstitution of the RIG-I pathway reveals a signaling role of unanchored polyubiquitin chains in innate immunity. Cell (2010) 141(2):315-30. doi:10.1016/j.cell. 2010.03.029

29. Maharaj NP, Wies E, Stoll A, Gack MU. Conventional protein kinase C-alpha (PKC-alpha) and PKC-beta negatively regulate RIG-I antiviral signal transduction. J Virol (2012) 86(3):1358-71. doi:10.1128/JVI. 06543-11

30. Oshiumi H, Miyashita M, Matsumoto M, Seya T. A distinct role of Riplet-mediated K63-Linked polyubiquitination of the RIG-I repressor domain in human antiviral innate immune responses. PLoS Pathog (2013) 9(8):e1003533. doi:10.1371/journal.ppat.1003533

31. Wies E, Wang MK, Maharaj NP, Chen K, Zhou S, Finberg RW, et al. Dephosphorylation of the RNA sensors RIG-I and MDA5 by the phosphatase 
PP1 is essential for innate immune signaling. Immunity (2013) 38(3):437-49. doi:10.1016/j.immuni.2012.11.018

32. Liu HM, Loo YM, Horner SM, Zornetzer GA, Katze MG, Gale M Jr. The mitochondrial targeting chaperone 14-3-3epsilon regulates a RIG-I translocon that mediates membrane association and innate antiviral immunity. Cell Host Microbe (2012) 11(5):528-37. doi:10.1016/j.chom.2012. 04.006

33. Wu B, Hur S. How RIG-I like receptors activate MAVS. Curr Opin Virol (2015) 12:91-8. doi:10.1016/j.coviro.2015.04.004

34. Matsumoto M, Oshiumi H, Seya T. Antiviral responses induced by the TLR3 pathway. Rev Med Virol (2011) 21(2):67-77. doi:10.1002/rmv.680

35. Hyun J, Kanagavelu S, Fukata M. A unique host defense pathway: TRIF mediates both antiviral and antibacterial immune responses. Microbes Infect (2013) 15(1):1-10. doi:10.1016/j.micinf.2012.10.011

36. Meylan E, Burns K, Hofmann K, Blancheteau V, Martinon F, Kelliher M, et al. RIP1 is an essential mediator of Toll-like receptor 3-induced NF-kappa B activation. Nat Immunol (2004) 5(5):503-7. doi:10.1038/ni1061

37. Estornes Y, Toscano F, Virard F, Jacquemin G, Pierrot A, Vanbervliet B, et al. dsRNA induces apoptosis through an atypical death complex associating TLR3 to caspase-8. Cell Death Differ (2012) 19(9):1482-94. doi:10.1038/ cdd.2012.22

38. Balachandran S, Thomas E, Barber GN. A FADD-dependent innate immune mechanism in mammalian cells. Nature (2004) 432(7015):401-5. doi:10.1038/nature03124

39. Lalani AI, Luo C, Han Y, Xie P. TRAF3: a novel tumor suppressor gene in macrophages. Macrophage (Houst) (2015) 2:e1009. doi:10.14800/ macrophage.1009

40. Gori-Savellini G, Valentini M, Cusi MG. Toscana virus NSs protein inhibits the induction of type I interferon by interacting with RIG-I. J Virol (2013) 87(12):6660-7. doi:10.1128/JVI.03129-12

41. Gori Savellini G, Gandolfo C, Cusi MG. Truncation of the C-terminal region of Toscana virus NSs protein is critical for interferon-beta antagonism and protein stability. Virology (2015) 486:255-62. doi:10.1016/j.virol.2015. 09.021

42. Zhu Z, Wang G, Yang F, Cao W, Mao R, Du X, et al. Foot-and-mouth disease virus viroporin $2 \mathrm{~B}$ antagonizes RIG-I mediated antiviral effects by inhibition of its protein expression. J Virol (2016). doi:10.1128/JVI.01310-16

43. Hou Z, Zhang J, Han Q, Su C, Qu J, Xu D, et al. Hepatitis B virus inhibits intrinsic RIG-I and RIG-G immune signaling via inducing miR146a. Sci Rep (2016) 6:26150. doi:10.1038/srep26150

44. Chan YK, Gack MU. A phosphomimetic-based mechanism of dengue virus to antagonize innate immunity. Nat Immunol (2016) 17(5):523-30. doi:10.1038/ni.3393

45. Miyashita M, Oshiumi H, Matsumoto M, Seya T. DDX60, a DEXD/H box helicase, is a novel antiviral factor promoting RIG-I-like receptor-mediated signaling. Mol Cell Biol (2011) 31(18):3802-19. doi:10.1128/MCB. 01368-10

46. Oshiumi H, Miyashita M, Okamoto M, Morioka Y, Okabe M, Matsumoto $\mathrm{M}$, et al. DDX60 is involved in RIG-I-dependent and independent antiviral responses, and its function is attenuated by virus-induced EGFR activation. Cell Rep (2015) 11(8):1193-207. doi:10.1016/j.celrep.2015.04.047

47. Schoggins JW, Wilson SJ, Panis M, Murphy MY, Jones CT, Bieniasz P, et al. A diverse range of gene products are effectors of the type I interferon antiviral response. Nature (2011) 472(7344):481-5. doi:10.1038/nature09907

48. Goubau D, van der Veen AG, Chakravarty P, Lin R, Rogers N, Rehwinkel J, et al. Mouse superkiller-2-like helicase DDX60 is dispensable for type I IFN induction and immunity to multiple viruses. Eur J Immunol (2015) 45(12):3386-403. doi:10.1002/eji.201545794

49. He Z, Zhu X, Wen W, Yuan J, Hu Y, Chen J, et al. Dengue virus subverts host innate immunity by targeting adaptor protein MAVS. J Virol (2016) 90(16):7219-30. doi:10.1128/JVI.00221-16

50. Dong J, Xu S, Wang J, Luo R, Wang D, Xiao S, et al. Porcine reproductive and respiratory syndrome virus $3 \mathrm{C}$ protease cleaves the mitochondrial antiviral signalling complex to antagonize IFN-beta expression. J Gen Virol (2015) 96(10):3049-58. doi:10.1099/jgv.0.000257

51. Li XD, Sun L, Seth RB, Pineda G, Chen ZJ. Hepatitis C virus protease NS3/4A cleaves mitochondrial antiviral signaling protein off the mitochondria to evade innate immunity. Proc Natl Acad Sci U S A (2005) 102(49):17717-22. doi:10.1073/pnas.0508531102
52. Ferreira AR, Magalhaes AC, Camoes F, Gouveia A, Vieira M, Kagan JC, et al. Hepatitis C virus NS3-4A inhibits the peroxisomal MAVS-dependent antiviral signalling response. J Cell Mol Med (2016) 20(4):750-7. doi:10.1111/ jcmm. 12801

53. Huang C, Du Y, Yu Z, Zhang Q, Liu Y, Tang J, et al. Highly pathogenic porcine reproductive and respiratory syndrome virus Nsp4 cleaves VISA to impair antiviral responses mediated by RIG-I-like receptors. Sci Rep (2016) 6:28497. doi:10.1038/srep28497

54. Cao L, Ge X, Gao Y, Herrler G, Ren Y, Ren X, et al. Porcine epidemic diarrhea virus inhibits dsRNA-induced interferon-beta production in porcine intestinal epithelial cells by blockade of the RIG-I-mediated pathway. Virol J (2015) 12:127. doi:10.1186/s12985-015-0345-x

55. Shi CS, Qi HY, Boularan C, Huang NN, Abu-Asab M, Shelhamer JH, et al. SARS-coronavirus open reading frame-9b suppresses innate immunity by targeting mitochondria and the MAVS/TRAF3/TRAF6 signalosome. J Immunol (2014) 193(6):3080-9. doi:10.4049/jimmunol.1303196

56. You F, Sun H, Zhou X, Sun W, Liang S, Zhai Z, et al. PCBP2 mediates degradation of the adaptor MAVS via the HECT ubiquitin ligase AIP4. Nat Immunol (2009) 10(12):1300-8. doi:10.1038/ni.1815

57. Hyun J, Ramos JC, Toomey N, Balachandran S, Lavorgna A, Harhaj E, et al. Oncogenic human T-cell lymphotropic virus type 1 tax suppression of primary innate immune signaling pathways. J Virol (2015) 89(9):4880-93. doi:10.1128/JVI.02493-14

58. Lui PY, Wong LY, Fung CL, Siu KL, Yeung ML, Yuen KS, et al. Middle east respiratory syndrome coronavirus $\mathrm{M}$ protein suppresses type I interferon expression through the inhibition of TBK1-dependent phosphorylation of IRF3. Emerg Microbes Infect (2016) 5:e39. doi:10.1038/emi. 2016.33

59. Siu KL, Chan CP, Kok KH, Chiu-Yat Woo P, Jin DY. Suppression of innate antiviral response by severe acute respiratory syndrome coronavirus $\mathrm{M}$ protein is mediated through the first transmembrane domain. Cell Mol Immunol (2014) 11(2):141-9. doi:10.1038/cmi.2013.61

60. Cramer P, Muller CW. A firm hand on NFkappaB: structures of the IkappaBalpha-NFkappaB complex. Structure (1999) 7(1):R1-6. doi:10.1016/ S0969-2126(99)80002-1

61. Yamamoto Y, Gaynor RB. IkappaB kinases: key regulators of the NF-kappaB pathway. Trends Biochem Sci (2004) 29(2):72-9. doi:10.1016/j.tibs.2003. 12.003

62. Kroll M, Margottin F, Kohl A, Renard P, Durand H, Concordet JP, et al. Inducible degradation of IkappaBalpha by the proteasome requires interaction with the F-box protein h-betaTrCP. J Biol Chem (1999) 274(12):7941-5. doi:10.1074/jbc.274.12.7941

63. Chen LF, Greene WC. Shaping the nuclear action of NF-kappaB. Nat Rev Mol Cell Biol (2004) 5(5):392-401. doi:10.1038/nrm1368

64. Huang L, Liu Q, Zhang L, Zhang Q, Hu L, Li C, et al. Encephalomyocarditis virus $3 \mathrm{C}$ protease relieves TRAF family member-associated NF-kappaB activator (TANK) inhibitory effect on TRAF6-mediated NF-kappaB signaling through cleavage of TANK. J Biol Chem (2015) 290(46):27618-32. doi:10.1074/jbc.M115.660761

65. Wang D, Fang L, Shi Y, Zhang H, Gao L, Peng G, et al. Porcine epidemic diarrhea virus $3 \mathrm{C}$-like protease regulates its interferon antagonism by cleaving NEMO. J Virol (2016) 90(4):2090-101. doi:10.1128/JVI.02514-15

66. Wang D, Fang L, Wei D, Zhang H, Luo R, Chen H, et al. Hepatitis A virus $3 \mathrm{C}$ protease cleaves NEMO to impair induction of beta interferon. J Virol (2014) 88(17):10252-8. doi:10.1128/JVI.00869-14

67. Wang D, Fang L, Li K, Zhong H, Fan J, Ouyang C, et al. Foot-and-mouth disease virus $3 \mathrm{C}$ protease cleaves NEMO to impair innate immune signaling. J Virol (2012) 86(17):9311-22. doi:10.1128/JVI.00722-12

68. Di Fiore IJ, Pane JA, Holloway G, Coulson BS. NSP1 of human rotaviruses commonly inhibits NF-kappaB signalling by inducing beta-TrCP degradation. J Gen Virol (2015) 96(Pt 7):1768-76. doi:10.1099/vir.0.000093

69. Fitzgerald KA, McWhirter SM, Faia KL, Rowe DC, Latz E, Golenbock DT, et al. IKKepsilon and TBK1 are essential components of the IRF3 signaling pathway. Nat Immunol (2003) 4(5):491-6. doi:10.1038/ni921

70. Sharma S, tenOever BR, Grandvaux N, Zhou GP, Lin R, Hiscott J. Triggering the interferon antiviral response through an IKK-related pathway. Science (2003) 300(5622):1148-51. doi:10.1126/science.1081315

71. Hacker H, Karin M. Regulation and function of IKK and IKK-related kinases. Sci STKE (2006) 2006(357):re13. doi:10.1126/stke.3572006re13 
72. Ikeda F, Hecker CM, Rozenknop A, Nordmeier RD, Rogov V, Hofmann K, et al. Involvement of the ubiquitin-like domain of TBK1/IKK-i kinases in regulation of IFN-inducible genes. EMBO J (2007) 26(14):3451-62. doi:10.1038/ sj.emboj.7601773

73. Verhelst K, Verstrepen L, Carpentier I, Beyaert R. IkappaB kinase epsilon (IKKepsilon): a therapeutic target in inflammation and cancer. Biochem Pharmacol (2013) 85(7):873-80. doi:10.1016/j.bcp.2013.01.007

74. Weil R, Laplantine E, Genin P. Regulation of TBK1 activity by optineurin contributes to cell cycle-dependent expression of the interferon pathway. Cytokine Growth Factor Rev (2016) 29:23-33. doi:10.1016/j.cytogfr.2016. 03.001

75. Zhao W. Negative regulation of TBK1-mediated antiviral immunity. FEBS Lett (2013) 587(6):542-8. doi:10.1016/j.febslet.2013.01.052

76. Honda K, Taniguchi T. IRFs: master regulators of signalling by toll-like receptors and cytosolic pattern-recognition receptors. Nat Rev Immunol (2006) 6(9):644-58. doi:10.1038/nri1900

77. Tamura T, Yanai H, Savitsky D, Taniguchi T. The IRF family transcription factors in immunity and oncogenesis. Annu Rev Immunol (2008) 26:535-84. doi:10.1146/annurev.immunol.26.021607.090400

78. Ikushima H, Negishi $H$, Taniguchi T. The IRF family transcription factors at the interface of innate and adaptive immune responses. Cold Spring Harb Symp Quant Biol (2013) 78:105-16. doi:10.1101/sqb.2013.78.020321

79. Yoneyama M, Suhara W, Fukuhara Y, Fukuda M, Nishida E, Fujita T. Direct triggering of the type I interferon system by virus infection: activation of a transcription factor complex containing IRF-3 and CBP/p300. EMBO J (1998) 17(4):1087-95. doi:10.1093/emboj/17.4.1087

80. Yang H, Ma G, Lin CH, Orr M, Wathelet MG. Mechanism for transcriptional synergy between interferon regulatory factor (IRF)-3 and IRF-7 in activation of the interferon- $\beta$ gene promoter. Eur J Biochem (2004) 271(18):3693-703. doi:10.1111/j.1432-1033.2004.04310.x

81. Yang Y, Ye F, Zhu N, Wang W, Deng Y, Zhao Z, et al. Middle East respiratory syndrome coronavirus ORF4b protein inhibits type I interferon production through both cytoplasmic and nuclear targets. Sci Rep (2015) 5:17554. doi:10.1038/srep17554

82. Christensen MH, Jensen SB, Miettinen JJ, Luecke S, Prabakaran T, Reinert LS, et al. HSV-1 ICP27 targets the TBK1-activated STING signalsome to inhibit virus-induced type I IFN expression. EMBO J (2016) 35(13):1385-99. doi:10.15252/embj.201593458

83. Zhang D, Su C, Zheng C. Herpes simplex virus 1 serine protease VP24 blocks the DNA-sensing signal pathway by abrogating activation of interferon regulatory factor 3. J Virol (2016) 90(12):5824-9. doi:10.1128/JVI. 00186-16

84. Dalrymple NA, Cimica V, Mackow ER. Dengue virus NS proteins inhibit RIG-I/MAVS signaling by blocking TBK1/IRF3 phosphorylation: dengue virus serotype $1 \mathrm{NS} 4 \mathrm{~A}$ is a unique interferon-regulating virulence determinant. MBio (2015) 6(3):e515-53. doi:10.1128/mBio.00553-15

85. Ding Z, Fang L, Jing H, Zeng S, Wang D, Liu L, et al. Porcine epidemic diarrhea virus nucleocapsid protein antagonizes beta interferon production by sequestering the interaction between IRF3 and TBK1. J Virol (2014) 88(16):8936-45. doi:10.1128/JVI.00700-14

86. Diani E, Avesani F, Bergamo E, Cremonese G, Bertazzoni U, Romanelli MG. HTLV-1 Tax protein recruitment into IKKepsilon and TBK1 kinase complexes enhances IFN-I expression. Virology (2015) 476:92-9. doi:10.1016/j. virol.2014.12.005

87. Yuen CK, Chan CP, Fung SY, Wang PH, Wong WM, Tang HM, et al. Suppression of type I interferon production by human T-cell leukemia virus type 1 oncoprotein tax through inhibition of IRF3 phosphorylation. J Virol (2016) 90(8):3902-12. doi:10.1128/JVI.00129-16

88. Masatani T, Ozawa M, Yamada K, Ito N, Horie M, Matsuu A, et al. Contribution of the interaction between the rabies virus $\mathrm{P}$ protein and I-kappa B kinase to the inhibition of type I IFN induction signalling. J Gen Virol (2016) 97(2):316-26. doi:10.1099/jgv.0.000362

89. Luo J, Fang L, Dong N, Fang P, Ding Z, Wang D, et al. Porcine deltacoronavirus (PDCoV) infection suppresses RIG-I-mediated interferon-beta production. Virology (2016) 495:10-7. doi:10.1016/j.virol.2016.04.025

90. Kotla S, Gustin KE. Proteolysis of MDA5 and IPS-1 is not required for inhibition of the type I IFN response by poliovirus. Virol J (2015) 12:158. doi:10.1186/s12985-015-0393-2
91. Huang F, Yang C, Yu W, Bi Y, Long F, Wang J, et al. Hepatitis E virus infection activates signal regulator protein alpha to down-regulate type I interferon. Immunol Res (2016) 64(1):115-22. doi:10.1007/s12026-0158729-y

92. Zhang R, Fang L, Wu W, Zhao F, Song T, Xie L, et al. Porcine bocavirus NP1 protein suppresses type I IFN production by interfering with IRF3 DNAbinding activity. Virus Genes (2016) 52(6):797-805. doi:10.1007/s11262-016$1377-\mathrm{z}$

93. Burysek L, Yeow WS, Lubyova B, Kellum M, Schafer SL, Huang YQ, et al. Functional analysis of human herpesvirus 8-encoded viral interferon regulatory factor 1 and its association with cellular interferon regulatory factors and p300. J Virol (1999) 73(9):7334-42.

94. Seo T, Lee D, Lee B, Chung JH, Choe J. Viral interferon regulatory factor 1 of Kaposi's sarcoma-associated herpesvirus (human herpesvirus 8) binds to, and inhibits transactivation of, CREB-binding protein. Biochem Biophys Res Commun (2000) 270(1):23-7. doi:10.1006/bbrc.2000.2393

95. Morin G, Robinson BA, Rogers KS, Wong SW. A rhesus rhadinovirus viral interferon (IFN) regulatory factor is virion associated and inhibits the early IFN antiviral response. J Virol (2015) 89(15):7707-21. doi:10.1128/ JVI.01175-15

96. Zhang Q, Shi K, Yoo D. Suppression of type I interferon production by porcine epidemic diarrhea virus and degradation of CREB-binding protein by nsp1. Virology (2016) 489:252-68. doi:10.1016/j.virol.2015.12.010

97. Bauhofer O, Summerfield A, Sakoda Y, Tratschin JD, Hofmann MA, Ruggli N. Classical swine fever virus Npro interacts with interferon regulatory factor 3 and induces its proteasomal degradation. J Virol (2007) 81(7):3087-96. doi:10.1128/JVI.02032-06

98. Seago J, Hilton L, Reid E, Doceul V, Jeyatheesan J, Moganeradj K, et al. The Npro product of classical swine fever virus and bovine viral diarrhea virus uses a conserved mechanism to target interferon regulatory factor-3. J Gen Virol (2007) 88(Pt 11):3002-6. doi:10.1099/vir.0.82934-0

99. Gottipati K, Holthauzen LM, Ruggli N, Choi KH. Pestivirus Npro directly interacts with interferon regulatory factor 3 (IRF3) monomer and dimer. J Virol (2016) 90(17):7740-7. doi:10.1128/JVI.00318-16

100. Lei X, Xiao X, Xue Q, Jin Q, He B, Wang J. Cleavage of interferon regulatory factor 7 by enterovirus 71 3C suppresses cellular responses. J Virol (2013) 87(3):1690-8. doi:10.1128/JVI.01855-12

101. Xiang Z, Liu L, Lei X, Zhou Z, He B, Wang J. 3C protease of enterovirus D68 inhibits cellular defense mediated by interferon regulatory factor 7. J Virol (2016) 90(3):1613-21. doi:10.1128/JVI.02395-15

102. Zhang BC, Zhou ZJ, Sun L. pol-miR-731, a teleost miRNA upregulated by megalocytivirus, negatively regulates virus-induced type I interferon response, apoptosis, and cell cycle arrest. Sci Rep (2016) 6:28354. doi:10.1038/ srep 28354

103. de Weerd NA, Nguyen T. The interferons and their receptors - distribution and regulation. Immunol Cell Biol (2012) 90(5):483-91. doi:10.1038/ icb.2012.9

104. Schindler C, Levy DE, Decker T. JAK-STAT signaling: from interferons to cytokines. J Biol Chem (2007) 282(28):20059-63. doi:10.1074/jbc. R700016200

105. Reich NC. STATs get their move on. JAKSTAT (2013) 2(4):e27080. doi: $10.4161 /$ jkst. 27080

106. Au-Yeung N, Mandhana R, Horvath CM. Transcriptional regulation by STAT1 and STAT2 in the interferon JAK-STAT pathway. JAKSTAT (2013) 2(3):e23931. doi:10.4161/jkst.23931

107. Fink K, Grandvaux N. STAT2 and IRF9: beyond ISGF3. JAKSTAT (2013) 2(4):e27521. doi:10.4161/jkst.27521

108. Porritt RA, Hertzog PJ. Dynamic control of type I IFN signalling by an integrated network of negative regulators. Trends Immunol (2015) 36(3):150-60. doi:10.1016/j.it.2015.02.002

109. Xia C, Vijayan M, Pritzl CJ, Fuchs SY, McDermott AB, Hahm B. Hemagglutinin of influenza A virus antagonizes type I interferon (IFN) responses by inducing degradation of type I IFN receptor 1. J Virol (2016) 90(5):2403-17. doi:10.1128/JVI.02749-15

110. Lubick KJ, Robertson SJ, McNally KL, Freedman BA, Rasmussen AL, Taylor RT, et al. Flavivirus antagonism of type I interferon signaling reveals prolidase as a regulator of IFNAR1 surface expression. Cell Host Microbe (2015) 18(1):61-74. doi:10.1016/j.chom.2015.06.007 
111. Guo L, Luo X, Li R, Xu Y, Zhang J, Ge J, et al. Porcine epidemic diarrhea virus infection inhibits interferon signaling by targeted degradation of STAT1. J Virol (2016) 90(18):8281-92. doi:10.1128/JVI.01091-16

112. Pisanelli G, Laurent-Rolle M, Manicassamy B, Belicha-Villanueva A, Morrison J, Lozano-Dubernard B, et al. La Piedad Michoacan Mexico Virus $\mathrm{V}$ protein antagonizes type I interferon response by binding STAT2 protein and preventing STATs nuclear translocation. Virus Res (2016) 213:11-22. doi:10.1016/j.virusres.2015.10.027

113. Hastings AK, Amato KR, Wen SC, Peterson LS, Williams JV. Human metapneumovirus small hydrophobic $(\mathrm{SH})$ protein downregulates type I IFN pathway signaling by affecting STAT1 expression and phosphorylation. Virology (2016) 494:248-56. doi:10.1016/j.virol.2016.04.022

114. Verweij MC, Wellish M, Whitmer T, Malouli D, Lapel M, Jonjic S, et al. Varicella viruses inhibit interferon-stimulated JAK-STAT signaling through multiple mechanisms. PLoS Pathog (2015) 11(5):e1004901. doi:10.1371/ journal.ppat.1004901

115. Kint J, Dickhout A, Kutter J, Maier HJ, Britton P, Koumans J, et al. Infectious bronchitis coronavirus inhibits STAT1 signaling and requires accessory proteins for resistance to type I interferon activity. J Virol (2015) 89(23):12047-57. doi:10.1128/JVI.01057-15

116. Wu J, Chen X, Ye H, Yao M, Li S, Chen L. Nonstructural protein (NS1) of human parvovirus B19 stimulates host innate immunity and blunts the exogenous type I interferon signaling in vitro. Virus Res (2016) 222:48-52. doi:10.1016/j.virusres.2016.06.004

117. Chaudhary V, Zhang S, Yuen KS, Li C, Lui PY, Fung SY, et al. Suppression of type I and type III IFN signalling by NSs protein of severe fever with thrombocytopenia syndrome virus through inhibition of STAT1 phosphorylation and activation. J Gen Virol (2015) 96(11):3204-11. doi:10.1099/jgv.0.000280

118. Ning YJ, Feng K, Min YQ, Cao WC, Wang M, Deng F, et al. Disruption of type I interferon signaling by the nonstructural protein of severe fever with thrombocytopenia syndrome virus via the hijacking of STAT2 and STAT1 into inclusion bodies. J Virol (2015) 89(8):4227-36. doi:10.1128/JVI.00154-15

119. Alexander WS, Hilton DJ. The role of suppressors of cytokine signaling (SOCS) proteins in regulation of the immune response. Annu Rev Immunol (2004) 22:503-29. doi:10.1146/annurev.immunol.22.091003.090312
120. Ilangumaran $S$, Ramanathan $S$, Rottapel R. Regulation of the immune system by SOCS family adaptor proteins. Semin Immunol (2004) 16(6):351-65. doi:10.1016/j.smim.2004.08.015

121. Sharma N, Kumawat KL, Rastogi M, Basu A, Singh SK. Japanese encephalitis virus exploits the microRNA-432 to regulate the expression of suppressor of cytokine signaling (SOCS) 5. Sci Rep (2016) 6:27685. doi:10.1038/ srep 27685

122. Choi EJ, Lee CH, Shin OS. Suppressor of cytokine signaling 3 expression induced by varicella-zoster virus infection results in the modulation of virus replication. Scand J Immunol (2015) 82(4):337-44. doi:10.1111/sji.12323

123. Zheng J, Yang P, Tang Y, Pan Z, Zhao D. Respiratory syncytial virus nonstructural proteins upregulate SOCS1 and SOCS3 in the different manner from endogenous IFN signaling. J Immunol Res (2015) 2015:738547. doi:10.1155/2015/738547

124. Walsh D, Mohr I. Viral subversion of the host protein synthesis machinery. Nat Rev Microbiol (2011) 9(12):860-75. doi:10.1038/nrmicro2655

125. Kint J, Langereis MA, Maier HJ, Britton P, van Kuppeveld FJ, Koumans J, et al. Infectious bronchitis coronavirus limits interferon production by inducing a host shutoff that requires accessory protein 5b. J Virol (2016) 90(16):7519-28. doi:10.1128/JVI.00627-16

126. Katsoulidis E, Kaur S, Platanias LC. Deregulation of interferon signaling in malignant cells. Pharmaceuticals (Basel) (2010) 3(2):406-18. doi:10.3390/ ph3020406

Conflict of Interest Statement: The authors declare that the research was conducted in the absence of any commercial or financial relationships that could be construed as a potential conflict of interest.

Copyright (c) 2016 Schulz and Mossman. This is an open-access article distributed under the terms of the Creative Commons Attribution License (CC BY). The use, distribution or reproduction in other forums is permitted, provided the original author(s) or licensor are credited and that the original publication in this journal is cited, in accordance with accepted academic practice. No use, distribution or reproduction is permitted which does not comply with these terms. 